Oвјестіvе: Cell adhesion molecules (intracellular adhesion molecule-1 (ICAM-1), vascular cell adhesion molecule-1 (VCAM-1)) and hyaluronic acid, markers of inflammation and fibrosis were monitored in hepatitis $C$ patients to determine whether changes in plasma levels, during antiviral treatment, can predict long-term response to therapy.

Metbods: In 55 patients with chronic hepatitis $\mathrm{C}$ virus (HCV), 33 treated with interferon (IFN) and 22 treated with IFN + ribavirin, sera was collected prior to treatment, at $3+6$ months of therapy and 6 months post-treatment. Levels of ICAM-1, VCAM-1 and hyaluronic acid were correlated with alanine aminotransferase levels, HCV-RNA-polymerase chain reaction status and histological fibrosis scoring.

Results: A decrease in ICAM-1 levels at 3 and 6 months of therapy, compared with pretreatment levels, was observed in responders to IFN + ribavirin therapy but this decrease in ICAM-1 levels was not evident following cessation of treatment. Hyaluronic acid levels, in both treatment groups, did not differ significantly between responders and non-responders. Hyaluronic acid levels did correlate, significantly, with degree of fibrosis whereas VCAM-1 levels were marginally increased only in patients with moderate (grade III) fibrosis.

Conclusions: Monitoring of VCAM-1 and hyaluronic acid, during antiviral therapy, does not differentiate between responders and non-responders. A decrease in ICAM-1 levels during IFN + ribavirin treatmment is associated with response to therapy, and its efficacy in predicting long-term response should be further substantiated.

Key words: HCV, Therapy, ICAM-1, VCAM-1, Hyaluronic acid

\section{Cell adhesion molecules and hyaluronic acid as markers of inflammation, fibrosis and response to antiviral therapy in chronic hepatitis $\mathrm{C}$ patients}

\author{
Esther Granot ${ }^{1, C A}$, Daniel Shouval ${ }^{2}$ and Yaffa Ashur ${ }^{2}$ \\ ${ }^{1}$ Pediatric Gastroenterology Unit, Pediatrics \\ Department and ${ }^{2}$ Liver Unit, Hadassah University \\ Hospital - Hebrew University-Hadassah Medical \\ School, P.O. Box 12000, Jerusalem 91120, Israel \\ ${ }^{\mathrm{CA}}$ Corresponding Author \\ Tel: +972 26777111 \\ Fax: +972 26434434 \\ E-mail: etgranot@md2.huji.ac.il
}

\section{Introduction}

Leukocyte adhesion molecules, among them intracellular adhesion molecule-1 (ICAM-1) and vascular cell adhesion molecule-1 (VCAM-1), play an integral role in infiltration, activation and binding of effector cells to tissues. During inflammation, activated vascular endothelial cells and other cell types express various adhesion molecules that facilitate binding of circulating leukocytes. Serum concentrations of these circulating adhesion molecules are thought to reflect the degree of this activation. These adhesion molecules also play a major role in mediating tissue damage during allograft rejection. ${ }^{1-4}$ In the liver of healthy controls, there is low basal expression of ICAM-1 on endothelial cells (liver sinusoidal cells) and low basal expression of VCAM-1 on both sinusoidal and Kupffer cells. In disease states, expression of adhesion molecules is markedly enhanced and ICAM1 can be found also on hepatocytes and on bile duct epithelium. ${ }^{3}$ Levels of circulating ICAM- 1 and VCAM-1 are increased in patients with hemochromatosis, liver cirrhosis and hepatocellular carcinoma, and have been shown to be useful in determining the severity of liver disease and the degree of fibrosis. ${ }^{1}$ ICAM-1 expression on biliary epithelium occurs in both primary sclerosing cholangitis (PSC) and primary biliary cirrhosis (PBC), and while it is less frequently observed in PBC patients, in PSC its occurrence correlates with advanced stages of the disease. ${ }^{2}$ In patients with chronic hepatitis C, serum ICAM-1 levels are believed to correlate with degree of active inflammation, whereas VCAM-1 levels reflect degree of fibrosis. ${ }^{5}$

An additional marker of liver fibrosis is hyaluronic acid, a high molecular weight polysaccharide produced by mesenchymal cells that is widely distributed in connective tissues. In the liver, sinusoidal endothelial cells rapidly accumulate and degrade hyaluronic acid. ${ }^{6}$ Serum levels of hyaluronic acid are increased in 
the presence of liver fibrosis and, in patients with chronic hepatitis $\mathrm{C}$ virus (HCV), hyaluronic acid levels were useful in detecting unsuspected fibrosis. ${ }^{7,8}$ In HCV patients treated with interferon (IFN), response to therapy has been suggested to be associated with a decrease in ICAM-1 levels $;^{9-12}$ also, in patients in whom post-therapy biopsies demonstrated an improvement in the degree of fibrosis, a significant decrease in hyaluronic acid levels has been observed. ${ }^{13,14}$

Current recommendations regarding duration of therapy in HCV-infected subjects vary, depending on genotype level of viremia and type of therapy (IFN monotherapy versus IFN + ribavirin combination therapy), but optimal duration of therapy remains to be discerned. ${ }^{15}$ We therefore questioned whether monitoring of changes in ICAM-1, VCAM-1 and hyaluronic acid levels during IFN or IFN + ribavirin therapy can serve in evaluating efficacy of treatment, thereby obviating prolonged treatment periods in patients who are unlikely to respond.

\section{Patients and methods}

\section{Patients}

Patient charts were reviewed retrospectively, and the relevant clinical data were obtained. Thirty-three patients (Group 1) were treated with IFN-alpha monotherapy $\left(3 \times 10^{6}-6 \times 10^{6}\right.$ international units (i.u.) thrice weekly), and 22 patients (Group 2) were treated with combination therapy $\left(3 \times 10^{6}-6 \times 10^{6}\right.$ IFN-alpha i.u. thrice weekly and 600-1200 mg ribavirin daily). Patients were randomly assigned to receive either IFN monotherapy or combination (IFN + ribavirin) therapy. The mean age was $38.4 \pm 13.4$ years (Group 1, $40.5 \pm 15.0$ years; Group 2, $34.6 \pm$ 11.8 years) and the male to female ratio was $1.2: 1$. Duration of therapy was 6-12 months.

Pre-treatment liver biopsies were available in all patients and all had chronic non-cirrhotic hepatitis $\mathrm{C}$ related liver disease. Post-treatment liver biopsies were available in 18 patients who responded to therapy. Biopsies were grouped based on the degree of fibrosis according to the modified Knodell scale: ${ }^{16}$ stage 0 , no fibrosis; stage I + II (mild fibrosis), portal fibrosis with fibrous portal expansion, or periportal fibrosis with periportal expansion, or rare portal-portal septae; and stage III (moderate fibrosis), bridging fibrosis with modular architecture distortion. Patients with cirrhosis on biopsy were not included.

All patients were Caucasians, with no history of drug or alcohol abuse and no evidence for coinfection with hepatitis B or HIV.

Definition of response to therapy: 'Response to therapy was defined as seroconversion to HCV-RNApolymerase chain reaction (PCR) negativity'.

\section{Serum analysis}

Serum samples were obtained from 55 anti-HCV+ adult patients who were treated at the Liver Unit of the Hadassah Medical Center, Jerusalem during 1996-1999. Anti-HCV was determined by the Corzyme-Enzyme immunoassay and confirmed by the Abbott HCV-neutralization EIA test (Abbott Labs., North Chicago, IL, USA).

All serum samples were examined for ICAM-1 and VCAM-1 levels using a radial immunoassay technique (R+D System, Minneapolis, MN, USA). Hyaluronic acid was determined using an assay based on initial binding of hyaluronic acid by specific binding protein (Chigai HA Assay, Chugai Diagnostic; Reaada Medical Products Inc., CO, USA). Each assay was performed in duplicate. ICAM-1, VCAM-1 and hyaluronic acid levels were correlated with biochemical alanine aminotransferase (ALT), virological (HCV-RNA-PCR; Roche) and histological parameters (Kuodell score). Tests were performed pre-therapy, at 3 and 6 months of therapy, and 6 months post-therapy.

\section{Statistical analysis}

Results were evaluated by one-way analysis of variance, and correlation coefficients were calculated using the InStat program. $p<0.05$ was considered statistically significant.

\section{Results}

Patients were randomly assigned to two groups: Group 1 were treated with IFN-alpha monotherapy $(n=33)$, and Group 2 were treated with combination therapy, IFN-alpha and ribavirin $(n=22)$. Past history of blood

\begin{tabular}{|c|c|c|c|c|}
\hline & Pre-treatment & After 3 months therapy & After 6 months therapy & Follow-up \\
\hline IFN - responders $(n=10)$ & $115.5 \pm 15.1$ & $31.7 \pm 6.0$ & $53.9 \pm 24.1$ & $39.3 \pm 8.0$ \\
\hline IFN + ribavarin, responders $(n=9)$ & $182.8 \pm 19.6$ & $29.9 \pm 2.9$ & $26.1 \pm 2.5$ & $22.8 \pm 3.3$ \\
\hline IFN + ribavarin, non-responders $(n=13)$ & $212.3 \pm 36.2$ & $128.8 \pm 23.4$ & $133.8 \pm 19.6$ & $180.5 \pm 39.1$ \\
\hline
\end{tabular}

Data presented as mean $\pm \mathrm{SE}$. 
(A)

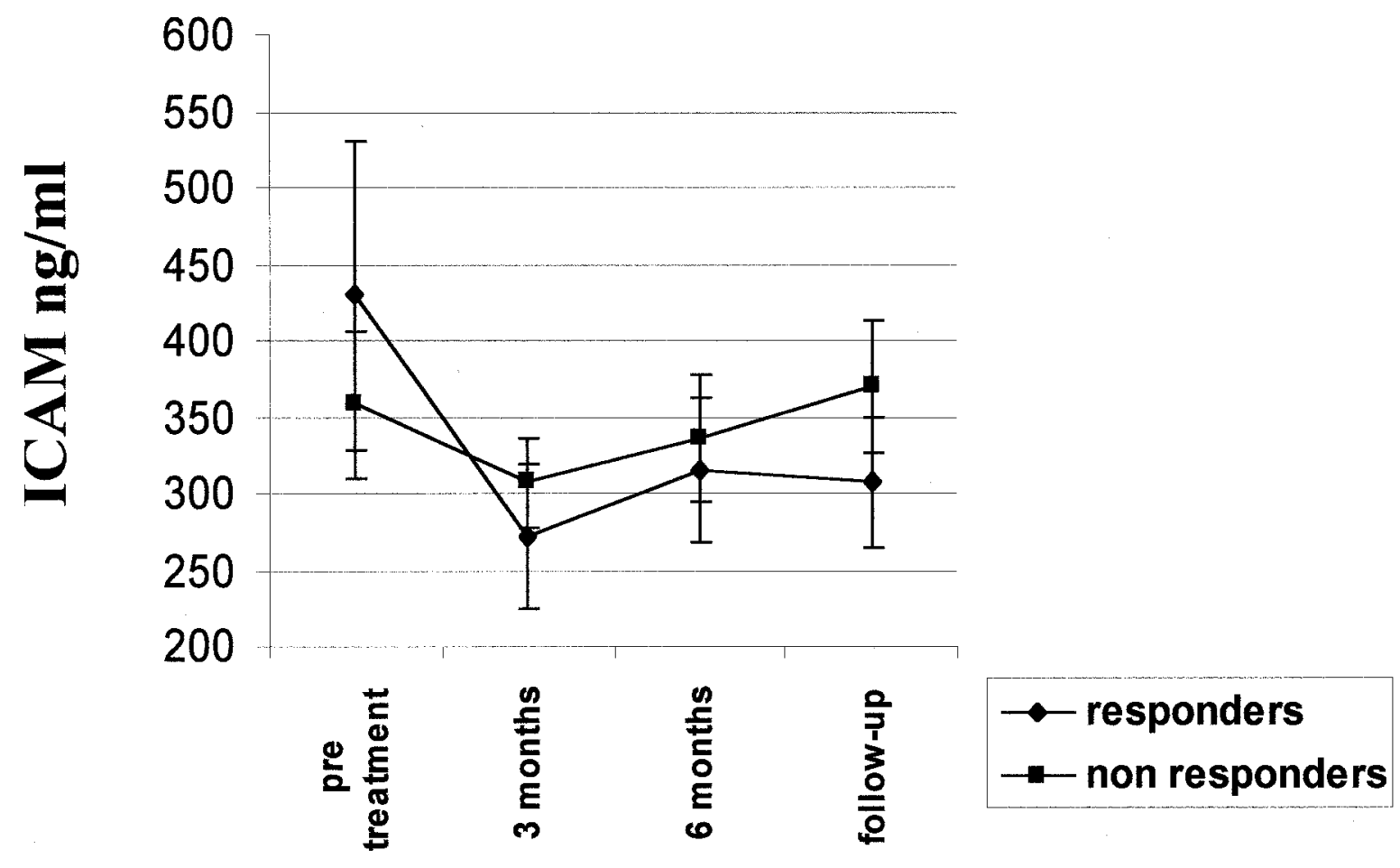

(B)

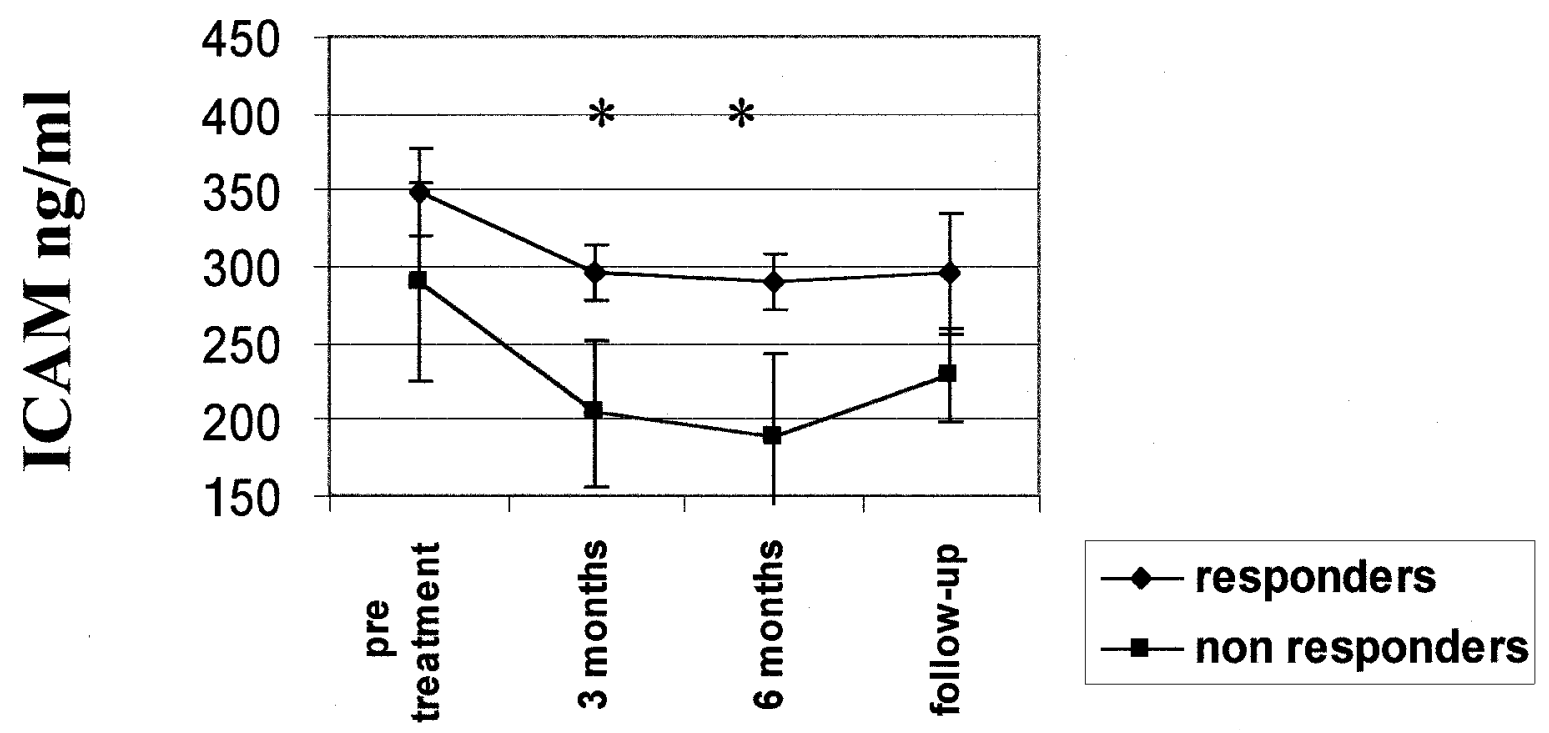

FIG. 1. (A) ICAM-1 levels $(\mathrm{ng} / \mathrm{ml})$ in responders $(n=10)$ versus non-responders $(n=23)$ to IFN-alpha monotherapy. (B) ICAM-1 levels $(\mathrm{ng} / \mathrm{ml})$ in responders $(n=9)$ versus non-responders $(n=13)$ to IFN-alpha + ribavirin combination therapy. (Results expressed as mean \pm SEM.)

transfusion, previous surgery, jaundice, family history of chronic liver disease (CLD) and hepatocellular carcinoma were also recorded with similar frequency in both groups. No significant difference was observed among groups in levels of hemoglobin, white blood cells, thrombocytes, prothrombin time, albumin, ALT, aspartate aminotransferase, alkaline phosphatase, and other markers for CLD. 


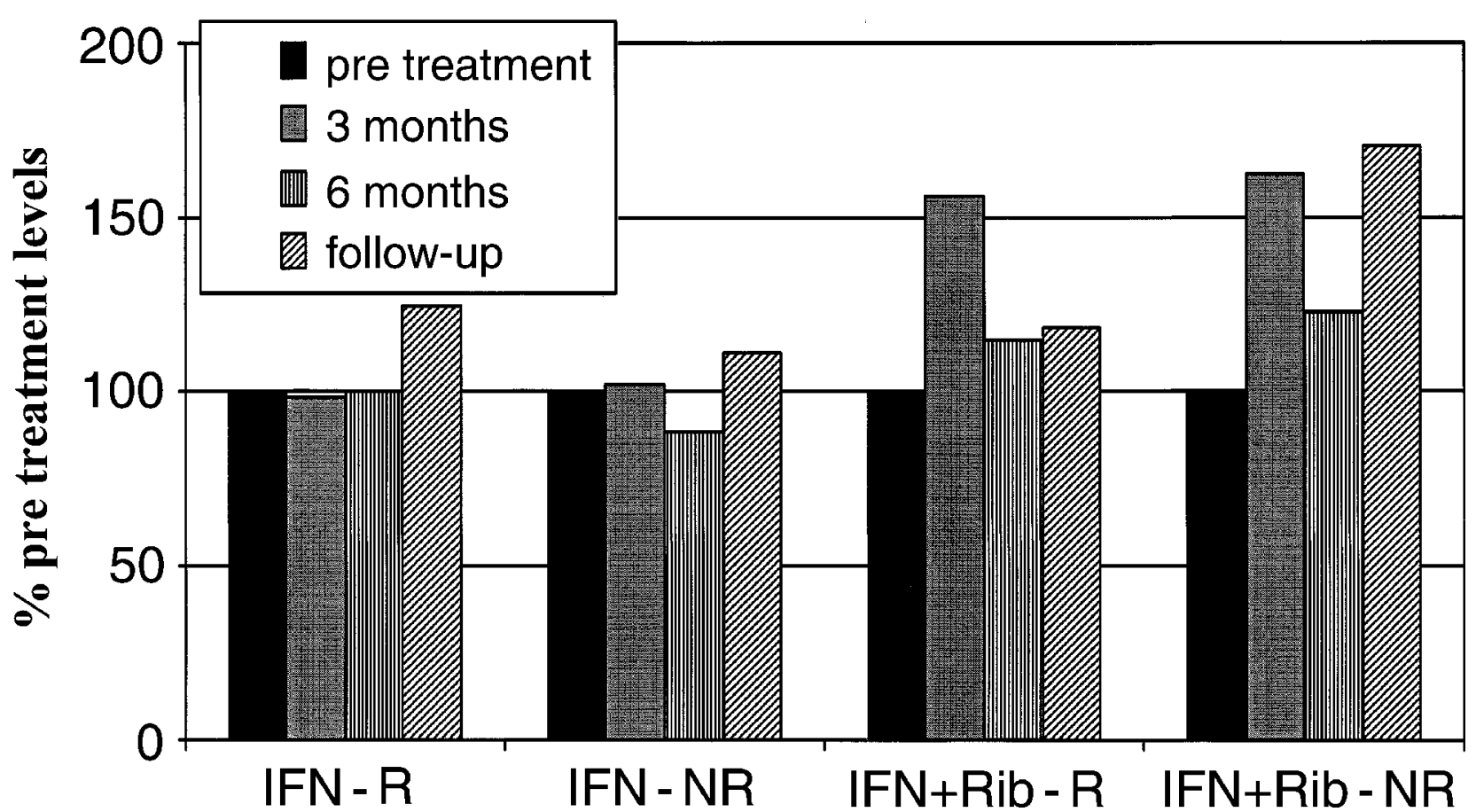

FIG. 2. VCAM-1 levels in responders (R) and non-responders (NR) to both IFN monotherapy (IFN) and IFN + ribavarin combination therapy (IFN + Rib). Results are expressed as percent of pre-treatment levels (pre-treatment levels $=100 \%$ ).

\section{ALT}

Pre-treatment levels did not differ between responders and non-responders in both treatment groups. However, ALT levels in responders were significantly lower $(p<0.05)$ after 3 and 6 months of therapy and at 6 months following cessation of therapy (Table 1).

\section{ICAM-1}

In patients receiving IFN monotherapy, differences between ICAM-1 levels in responders and nonresponders did not differ significantly at all time points studied (Fig. 1A). In patients receiving IFN + ribavirin therapy ICAM-1 levels in responders were significantly lower $(p<0.05)$ than levels in nonresponders at 3 and 6 months of therapy, while pretreatment levels and those 6 months after cessation of therapy, albeit lower in responders, did not differ significantly from those in non-responders (Fig. 1B).

\section{VCAM-1}

Levels did not differ significantly between responders and non-responders at all time points studied (Fig. 2). Changes in VCAM-1 levels did not correlate with ALT changes, both in responders and in non-responders. VCAM-1 levels in patients with moderate fibrosis $\left(\mathrm{F}_{3}\right)$ were higher than in patients with no fibrosis $\left(\mathrm{F}_{1}\right)$ but differences were only marginally significant $(p=$ 0.06).

\section{Hyaluronic acid}

In both the monotherapy and combination therapy groups, hyaluronic acid levels did not differ significantly between responders and nonresponders at all time points studied. Changes in VCAM-1 levels did not correlate with changes in hyaluronic acid levels both in responders and nonresponders.

Table 2. Fibrosis scoring versu ALT, ICAM-I, VCAM-I and hyaluronic acid

\begin{tabular}{|c|c|c|c|}
\hline $\begin{array}{l}M T=\text { Monotherapy } \\
C T=\text { Combination therapy }\end{array}$ & $\begin{array}{c}\text { Mild hepatitis } \\
(n=9 ; \mathrm{MT}=5 ; \mathrm{CT}=4)\end{array}$ & $\begin{array}{c}\text { Moderate to } \\
\text { severe hepatitis } \\
(n=54 ; \mathrm{MT}=29 ; \mathrm{CT}=25)\end{array}$ & $\begin{array}{c}\text { Active cirrhosis } \\
(n=10 ; \mathrm{MT}=6 ; \mathrm{CT}=4)\end{array}$ \\
\hline $\mathrm{ALT}(\mathrm{IU})$ & $81.8 \pm 10.1$ & $95.9 \pm 5.5$ & $67.8 \pm 7.5$ \\
\hline ICAM-I (ng/ml) & $214 \pm 84$ & $196 \pm 33$ & $200 \pm 70$ \\
\hline VCAM-I (ng/ml) & $872 \pm 193$ & $1023 \pm 73$ & $1340 \pm 144$ \\
\hline Hyaluronic acid (ng/ml) & $42.3 \pm 8.4$ & $62.5 \pm 4.5$ & $95.2 \pm 8.2$ \\
\hline
\end{tabular}

Data presented as mean $\pm \mathrm{SE}$. 


\section{Liver histology}

Table 2 summarizes the correlation between the degree of fibrosis $\left(F_{1}\right.$, no fibrosis; $F_{2}$, mild fibrosis; and $\mathrm{F}_{3}$, moderate fibrosis) with levels of ALT, ICAM-1, VCAM-1 and hyaluronic acid at the time of liver biopsy, regardless of response to therapy. Changes in ALT and ICAM-1 did not correlate with the F score (as expected). However, VCAM-1 levels were higher in the $F_{3}$ group than in the $F_{1}$ group, but this difference was only marginally significant $(p=0.06)$. Hyaluronic acid levels were significantly higher in the $F_{3}$ group as compared with the $\mathrm{F}_{1}$ and $\mathrm{F}_{2}$ groups $(p<0.01$ and $p$ $<0.05$ respectively).

\section{Discussion}

Optimal duration of antiviral therapy in chronic HCV remains an unsettled issue and recommendations vary depending on the protocols followed. ${ }^{15,17,18}$ Markers that may aid in prediction of response, and thereby obviate futile treatment in those patients who are unlikely to respond, are thus of utmost importance. Previous studies have suggested a role for serum hyaluronic acid determinations in monitoring of response to antiviral therapy. Yamada et al. ${ }^{13}$ assessed changes in serum hyaluronic acid in 35 patients treated with IFN. In complete responders, a significant decrease of serum hyaluronic acid levels was noted, in parallel with histological improvement, whereas in partial responders and non-responders this decrease in hyaluronic acid levels was not observed. A similar study by Ninomiya et al. ${ }^{14}$ substantiated the observation that hyaluronic acid is a useful marker of liver fibrosis in HCV patients but emphasized that it cannot differentiate sustained responders from relapsers or non-responders. In our patients, although hyaluronic acid levels did correlate with degree of fibrosis and levels measured were indeed higher in non-responders, during the course of therapy, in each treatment group, differences between responders and non-responders were not significant. Thus, monitoring of hyaluronic acid levels at initiation of antiviral therapy and during the course of therapy cannot serve in predicting response but is, indeed, a useful parameter in assessing liver fibrosis.

VCAM-1 levels have also been shown to reflect the degree of liver fibrosis. ${ }^{5,10}$ Although in our patients in whom biopsies demonstrated moderate fibrosis $\left(\mathrm{F}_{3}\right)$ the levels were higher than in $\mathrm{HCV}$ patients with no fibrosis, differences were only marginally significant. Furthermore, VCAM-1 levels during the course of therapy did not differ between responders and non-responders.

ICAM-1 levels, markers of liver inflammation, have been shown to remain elevated in $\mathrm{HCV}$ patients who do not respond to IFN therapy, whereas in patients with normal ALT levels following interferon administration, ICAM-1 levels have significantly decreased. $^{9-12}$ In our study, when analyzing the longitudinal course of ICAM-1 levels within each group of patients, a decrease in ICAM-1 levels at 3 and 6 months of therapy, compared with pretreatment levels, was observed in responders to therapy. This decrease was most notable, and reached statistical significance, only in the responders to combination (IFN + ribavirin) therapy. Yet, even in this group of responders, the decrease in ICAM-1 levels was not evident long term; 6 months following cessation of treatment, ICAM-1 levels did not differ significantly from pre-treatment levels (Fig. 1). Furthermore, in contrast with the observations of Baguasco et al. ${ }^{11}$ who noted higher basal pretreatment ICAM-1 levels in responders to therapy, compared with non-responders, in our patients pretreatment levels in responders and non-responders did not differ significantly.

Thus, although response to IFN + ribavirin antiviral therapy is associated with a transient decrease in ICAM-1 levels, further studies are needed to substantiate the role of ICAM-1 monitoring in predicting long-term response. As for the monitoring of VCAM-1 and hyaluronic acid during antiviral therapy, pretreatment levels and levels measured during the course of treatment cannot serve in predicting response to treatment, nor can their determination following cessation of therapy define responders from non-responders.

\section{References}

1. Stal P, Broome U, Schevnius A, Befrits R, et al. Kupffer cell iron overload induces intercellular adhesion molecule-1 expression in hepatocytes in genetic hemochromatosis. Hepatology 1995; 21: 1308-1316.

2. Nishimura Y, Takei Y, Kawano S, et al. The F[ab'(12 fragment of an anti ICAM-1 monoclonal antibody attenuates liver injury after orthotopic liver transplantation. Transplantation 1996; 61: 99-104.

3. Lang T, Krams SM, Villanueva JC, et al. Differential patterns of circulating adhesion molecule-1 and vascular adhesion molecule-1 during liver allograft rejection. Transplantation 1995; 59: 584-589.

4. Bloom S, Fleming K, Chapman R. Adhesion molecule expression in primary sclerosing cholangitis and primary biliary cirrhosis. Gut 1995; 36: 604-609.

5. Kaplanski G, Frananier C, Payan MJ, et al. Increased levels of soluble adhesion molecules in the serum of patients with hepatitis C. Correlation with cytokine concentrations and liver inflammation and fibrosis Dig Dis Sci 1997; 42: 2277-2284.

6. Engstrom-Laurent A, Loof L, Nyberg A, SchroderT. Increased serum levels of hyaluronate in liver disease. Hepatology 1985; 5: 638-642.

7. Ueno T, Inuzuka S, Tomimura T, et al. Serum hyaluronate reflects hepatic sinusoidal capillarization. Gastroenterology 1993; 105: 475-481.

8. Wong VS, Hughes V, Trulla A, et al. Serum hyaluronic acid is a useful marker of liver fibrosis in chronic hepatitis C virus infection. $J$ Viral Hepat 1998; 5: 187-192.

9. Gattoni A, Romano C, Cecere A, et al. Serum levels of soluble intercellular adhesion molecule 1 (sICAM-1) as a potential marker of disease activity and remission in patients with chronic hepatitis C. Paninerva Med 1997; 39: $256-262$.

10. Lolacono O, Garcia-Monzon C, Almasio $\mathrm{P}$, et al. Soluble adhesion molecules correlate with liver inflammation and fibrosis in chronic hepatitis C treated with interferon-alpha. Aliment Pharmacol Ther 1998; 12: 1091-1099.

11. Baguasco M, Riccio AM, Sinelli M. Serum intercellular adhesion molecule1 and long-term response to IFN-alpha $2 \mathrm{~B}$ therapy in chronic hepatitis C. J Interferon Cytokine Res 1998; 18: 963-966. 
12. Capra F, Demaria E, Lunardi C, et al. Serum level of soluble intercellular adhesion moelcule 1 in patients with chronic liver disease related to hepatitis C virus: a prognostic marker for responses to interferon treatment. J Infect Dis 2000; 181: 425-431.

13. Yamada M, Fuduka Y, Koyama Y, et al. Serum hyaluronic acid reflects the effect of interferon treatment on hepatic fibrosis in patients with chronic hepatitis C. J Gastroenterol Hepatol 1996; 11: 646-651.

14. Ninomiya T, Yoon S, Hayashi Y et al. Clinical significance of serum hyaluronic acid as a fibrosis marker in chronic hepatitis $\mathrm{C}$ patients treated with interferon-alpha: histological evaluation by a modified histological activity index scoring system.J Gastroenterol Hepatol 1998; 13: $68-74$.

15. Anonymous. EASL Consensus Panel International Consensus Conference on Hepatitis C. J Hepatol 1999; 30: 956-961.
16. Knodell RG, Ishak KG, Black WC, et al. Formulation and application of a numerical scoring system for assessing histological activity in asymptomatic chronic active hepatitis. Hepatology 1981; 1: 431-435.

17. Bacon BR. Available options for treatment of interferon nonresponders. Am J Med 1999; 107: 67S-70S.

18. Jacobson I. Management of interferon relapsers. Am J Med 1999; 107: 625-665.

\section{Received 19 June 2001}

Accepted 3 August 2001 


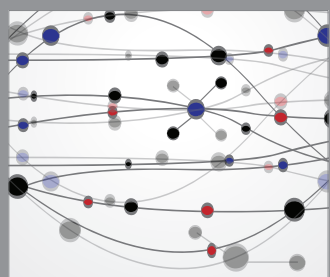

The Scientific World Journal
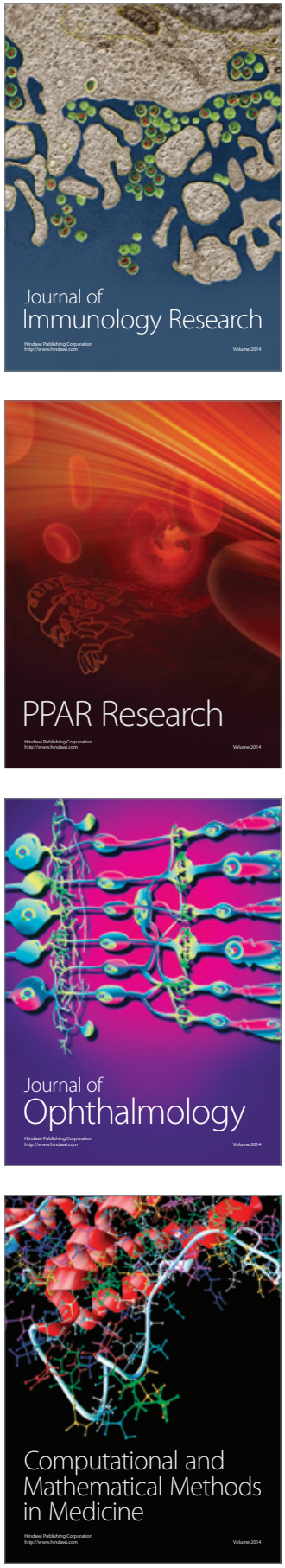

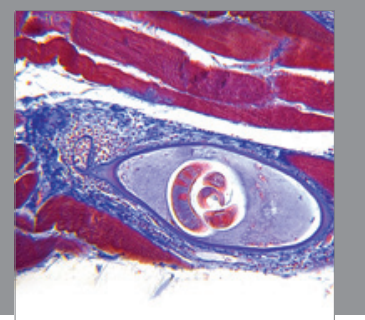

Gastroenterology

Research and Practice
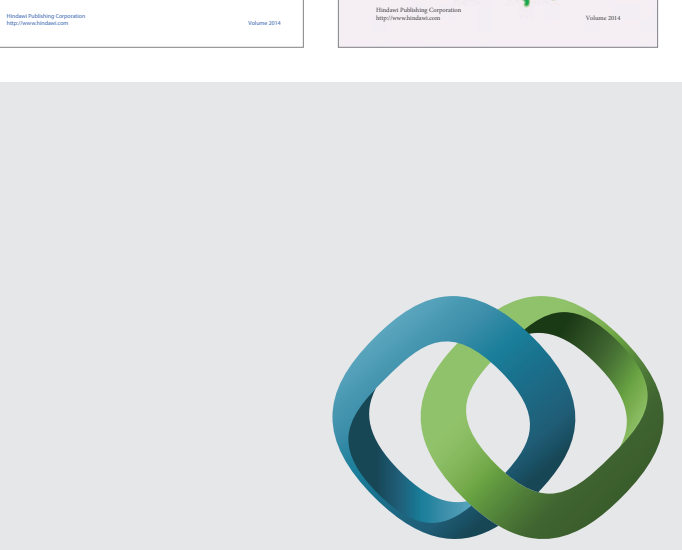

\section{Hindawi}

Submit your manuscripts at

http://www.hindawi.com
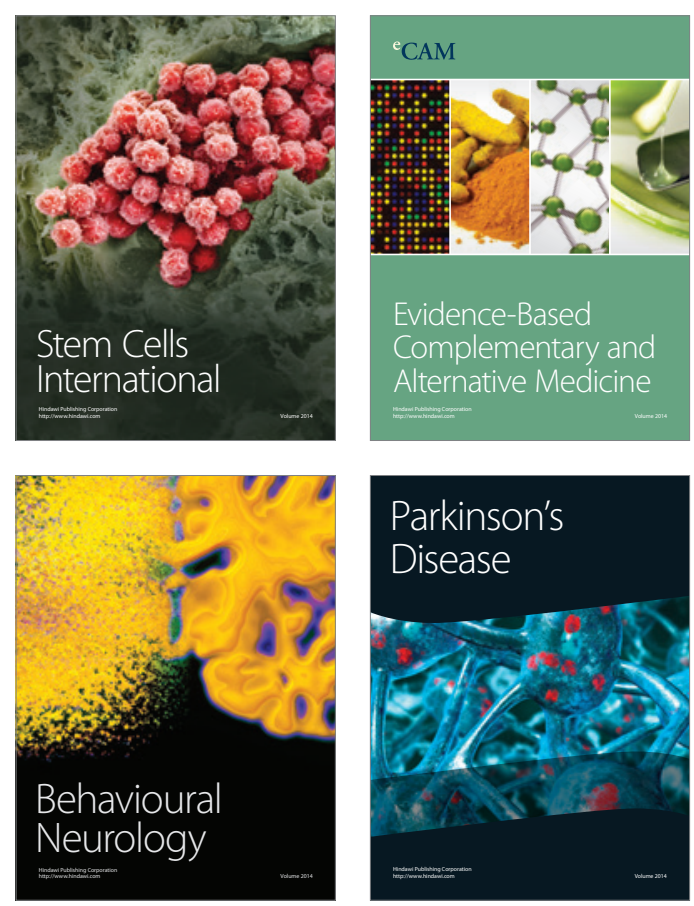

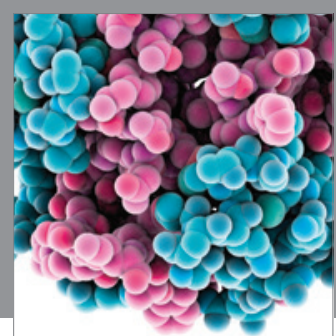

Journal of
Diabetes Research

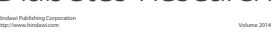

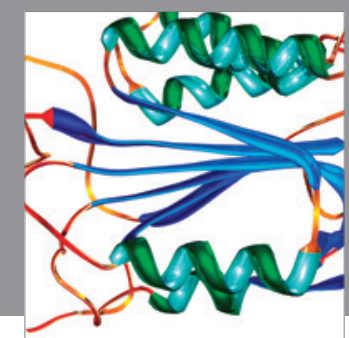

Disease Markers
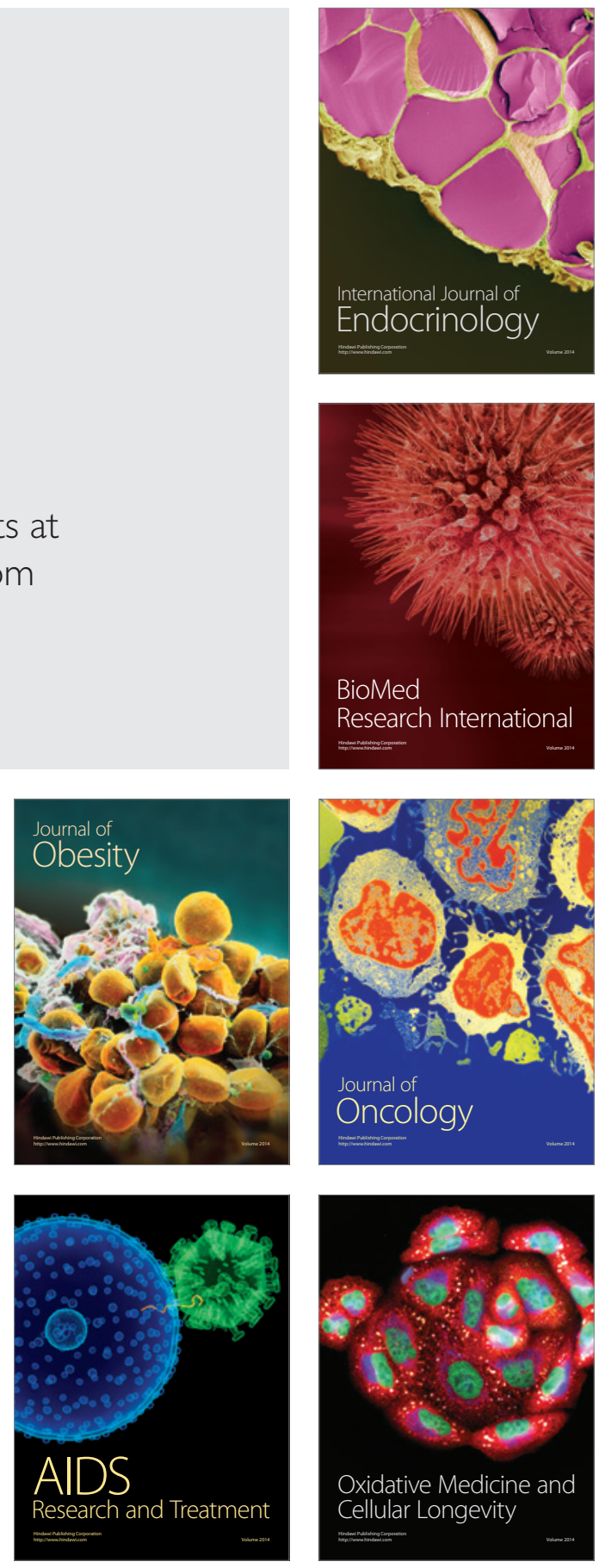